\title{
Imaging Radiation-Induced gamma H2AX Foci
}

\author{
L.S. Yasui \\ Department of Biological Sciences, Northern Illinois University, DeKalb, IL 60115
}

Cells respond to the induction of DNA double strand breaks (dsbs) (induced by ionizing radiation, DNA damaging drugs, apoptosis, meiotic recombination or $\mathrm{V}(\mathrm{D}) \mathrm{J}$ recombination) by phosphorylating a variant form of histone $\mathrm{H} 2 \mathrm{AX}$ around the DNA dsb in chromosome domains [1]. Several thousands of the phosphorylated H2AX molecules, called gamma H2AX, are clustered around the DNA dsb to form a spot or foci in the cell nucleus that can be detected by immunofluorescence staining methods [1,3]. Phosphorylation of H2AX by ATM, ATR or DNAPK [2], occurs within minutes of DNA dsb induction and it is one of the earliest cell signaling events in the DNA damage response pathway. This pathway leads to cell cycle checkpoint events and/or DNA damage repair [2]. The purpose of these studies is to investigate the role of gamma $\mathrm{H} 2 \mathrm{AX}$ foci in recruiting DNA damage repair molecules and the initiation of cell cycle checkpoints.

Challenges exist in the development of 3D imaging methods to clearly and accurately describe gamma H2AX foci formation. Methods used to quantify gamma H2AX by flow cytometry and western blotting do not elucidate differences in the structure that can be used to describe the functional significance of these foci in cells. For example, number of foci, and differences in size, shape, clustering and nuclear location may be altered depending upon cell type, type of irradiation or metabolic status of the cell. Clearly, confocal laser scanning microscopy (CLSM) methods offer optimal 3D imaging capabilities. Several cell fixation protocols were tested using human breast cancer cells, MCF-7. Fixation using 2\% formaldehyde in PBS resulted in optimal cell preservation of 3D shapes. A standard immunofluorescence staining protocol was used [1-3]. CLSM parameters were optimized. All images were scanned at 1024 X 1024 pixels without zoom using a Zeiss LSM 5 Pascal VarioTwo CLSM and Zeiss 510 software. Pinhole size set to 1 airy unit, and the software set to acquire images as 8 bit images using a Plan Neo 40X, NA 1.3 objective lens.

Several steps were tested to ensure that we acquired gamma H2AX foci image information that is high resolution, accurate and reliable. Calculations were performed to confirm digital image quality based on the Nyquist sampling theorem and an experimentally determined average cell nucleus size containing 60 X 60 pixels. The calculated dimension of each pixel was equal to $230 \mathrm{~nm}$ (close to the limit of resolution of the light microscope). This value is larger than the theoretical optimum ( 80 $\mathrm{nm}$ ) but the benefits of this under sampling were offset by reduced fluorescence bleaching and increased field of view. Further, profile analysis, thresholding, counter staining cells with propidium iodide and $3 \mathrm{D}$ intensity histogram analysis were all used as tools to discriminate gamma $\mathrm{H} 2 \mathrm{AX}$ foci in individual cells.

\section{References}

[1] E.P. Rogakou et al., J. Biol. Chem. 273 (1998) 5858.

[2] T. Stiff et al., Canc. Res. 64 (2004) 2390.

[3] L.S. Yasui. Int. J. Radiat. Biol. 80 (2004) 895. 

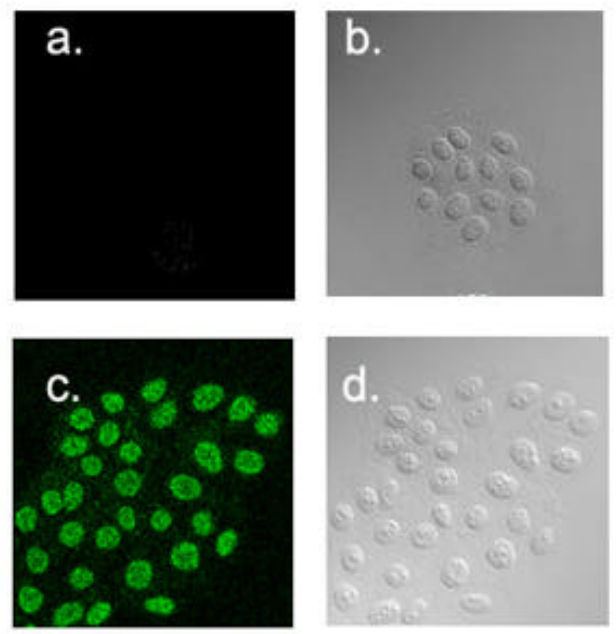

Fig.1 CLSM imaging of gamma H2AX foci in unirradiated MCF-7 cells $(\mathrm{a}, \mathrm{b})$ and MCF-7 cells irradiated with 10 Gy gamma rays.

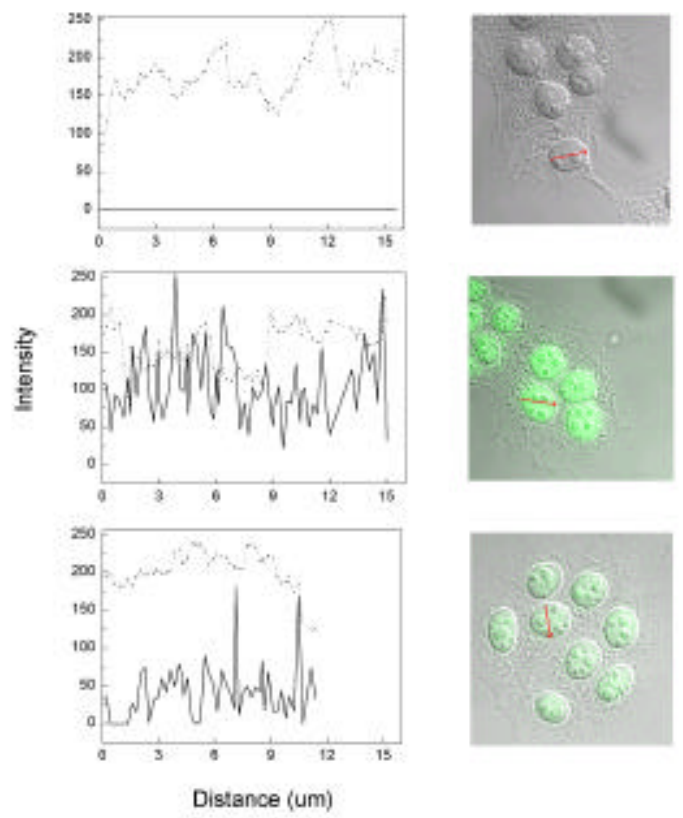

Fig. 3. Profile analysis of gamma $\mathrm{H} 2 \mathrm{AX}$ foci in irradiated (middle and bottom panels) and unirradiated MCF-7 cells (top panel).
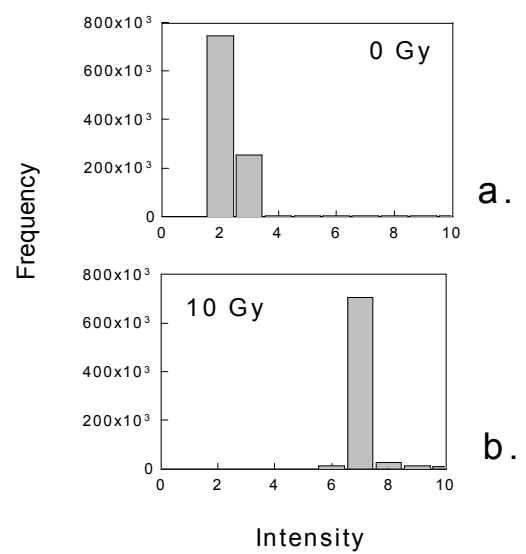

Fig. 2. Histogram analysis of irradiated (panel a) and unirradiated (panel b) MCF7 cells.

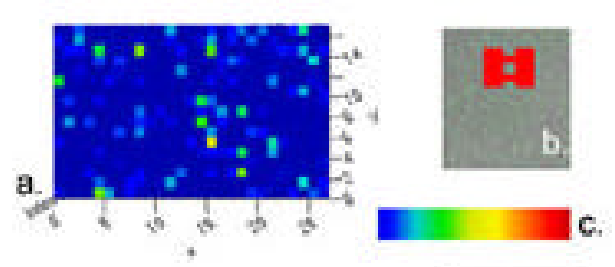

d.
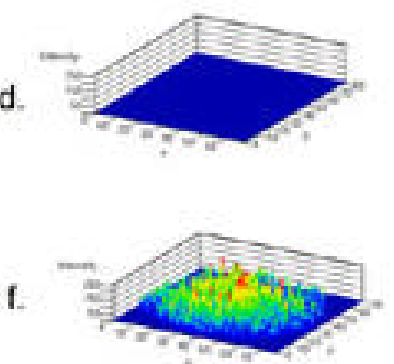

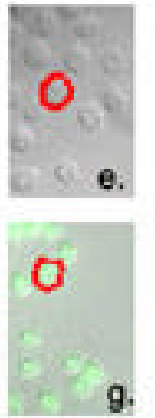

Fig. 4. Thresholding and 3D histogram analysis of gamma $\mathrm{H} 2 \mathrm{AX}$ foci in irradiated (bottom panel) and unirradiated MCF-7 cells (middle panel). Threshold was set by the background (top panel). 